\title{
Rezeption, Perzeption und Adaption: Erwartungen an die chinesische Rechtsmodernisierung 1904-1930
}

\author{
Von Robert Heuser*
}

\begin{abstract}
Between the 1870ies and the outbreak of the Pacific war in 1937 Japan and China undertook fundamental changes in their traditional legal systems by way of legislation strongly following continental Europen models. These changes were not based on the free choice of the political elites of these countries, but were suggested and induced by outside pressure. In this paper certain statements or commentaries of participants in the modernization process as it took place in China during the first third of the 20th century are presented in order to exemplify how the motives of law reform were understood, how the legal cultures involved were perceived, and how the problem of adaptation was tackled.
\end{abstract}

\section{A. Historisches Umfeld und Fragestellung}

Zwischen den 1870er Jahren und dem Ausbruch des Pazifischen Krieges 1937 fanden in Japan und China grundlegende Veränderungen der überlieferten Rechtssysteme durch eine Gesetzgebung statt, die sich an kontinental-europäischen Mustern orientierte. Diese Rechtsmodernisierung resultierte nicht oder kaum aus dem inneren Entwicklungsgang dieser Länder, sondern sie wurde maßgeblich von Außen veranlasst. Sie war die Folge der durch die industrielle Revolution gewonnenen technischen und militärischen Dominanz des Westens. Aus dieser Dominanz erwuchs der Kolonialismus zum einen als wirtschaftliches Interesse, zum andern als kultureller Imperialismus dergestalt, dass die Europäer nicht nur technisches Wissen nach Ostasien brachten, sondern auch ihren Normensystemen - Staats-, Rechts- und Religionssystemen - universelle Bedeutung beimaßen. Das herrschende Paradigma war das der „Zivilisierung“, also die Idee, dass diese Länder nicht ausreichend zivilisiert seien und es Aufgabe des Westens sei, sie nach dem westlichen Modell zu modernisieren. Andererseits nutzten reformgesinnte und sich gegenüber eindringenden sozialen und politischen Ideen Europas nicht gänzlich verschließende Eliten dieser Länder die durch die Konfrontation mit den westlichen Mächten herbeigeführte Lage, um als nötig empfundene institutionellen Veränderungen - unter Einschluss solche des Rechtssystems - zu propagieren und voranzutreiben. Dabei

* Prof. em. Dr. Robert Heuser, Team chinesische Rechtskultur, Philosophische Fakultät der Universität zu Köln-robert.heuser@uni-koeln.de. 
sollte dem westlichen Recht im Übergang vom traditionellen zum modernen Recht eine „Schlüsselrolle und wichtige Funktion zufallen“. ${ }^{1}$

Nachdem in einigen unglücklichen Vorfällen chinesische Untertanen durch Ausländer verletzt oder getötet worden waren, und die Ausländer das chinesische Strafrecht als Erfolgsstrafrecht ohne ausreichende Berücksichtigung der Schuldfrage und sonstige archaische Elemente des materiellen und formellen Strafrechts (wie kollektive Verantwortlichkeit, Prügelstrafe und Geständniserpressung durch Folter) wahrgenommen hatten, verlangten sie Gewährung von Exterritorialität für ihre in China tätigen Staatsangehörigen, also die Anwendung des eigenen Rechts im Verfahren vor den eigenen diplomatischen Vertretungen.

Diese Privilegien wurden in zahlreichen völkerrechtlichen Verträgen während der zweiten Hälfte des 19. Jh. von der Qing-Regierung zugesichert. So heißt es im britisch-chinesischen Vertrag von 1858: „Sämtliche Fragen betreffend Vermögens- und Personenrechte, die sich zwischen britischen Untertanen ergeben, unterliegen der Gerichtsbarkeit der britischen Behörden. - Gegen britische Untertanen, die in China eine Straftat begehen, wird ein Strafverfahren vor den britischen Konsul nach britischem Recht durgeführt." Auch wenn die QingRegierung Konzepten wie „Souveränität“ und „territoriale Integrität“" zunächst wenig Bedeutung beimaß, so stießen die Privilegien der Ausländer doch zunehmend auf den Widerstand einer sich der eigenen Nationalität bewusst werdenden Beamtenschaft und Bevölkerung, wie dies besonders im sog. Boxer-Aufstand von 1900 manifest wurde, und die Abschaffung der „ungleichen Verträge“" wurde zu einem Hauptanliegen der chinesischen Außenpolitik. Von europäischer Seite wurde ein Entgegenkommen in Aussicht gestellt, wenn China sein Rechtssystem nach europäischem Muster neu gestalte. Der sich so ergebende Zusammenhang von Rückgabe von Souveränitätsrechten und Rechtsmodernisierung kam im chinesisch-britischen Handelsvertrag von 1902 in direkter Weise zum Ausdruck: „China having expressed a strong desire to reform her judicial system and to bring it into accord with that of Western nations, Great Britain agrees to give every assistance to such reforms, and she will be prepared to relinquish her exterritorial rights when she is satisfied that the State of Chinese laws...warrant her in so doing“ (Art. 12). Dies war, nachdem von den höchsten Rängen der chinesischen Bürokratie bereits 1901 der Anstoß für Reformen erfolgt war, der unmittelbare Anlass, dass die Qing-Regierung 1904 eine „Behörde für die Zusammenstellung der Gesetze“ (法律编纂 馆) unter Leitung eines in der Geschichte des chinesischen Rechts intensiv bewanderten Beamten des Strafministeriums, Shen Jiaben (1840-1913), und dem britisch ausgebildeten und als erster chinesischer Barrister in Hongkong tätig gewesenen Wu Tingfan (1842-1922) etablierte und damit begonnen wurde, ausländische Gesetze zu übersetzen und Gesetzesentwürfe gemäß diesem Muster zu formulieren und zunächst nur teilweise, dann zunehmend mehr in Kraft zu setzen. Formal abgeschlossen wurde der Modernisierungsvorgang 1930 mit dem

1 Etwa Zhang Jinfan, 综论中国法制的近代化 (Zusammenfassende Erörterung der Modernisierung des chinesischen Rechtssystems), in: 政法论坛 , 2004, Nr. 1, S. 3 ff. 
Erlass des ZGB. ${ }^{2} \mathrm{Zu}$ Beginn dieses Geschehens sah der in alten und neuen europäischen Sprachen und in der europäischen Kultur bewanderte Gu Hongming (1857-1928), ein Mitarbeiter von Zhang Zhidong, „die Tragik der gegenwärtigen Lage in China darin, dass, während die ganze chinesische Nation entschlossen ist, ihre eigene Kultur wegzuwerfen und die Kultur des modernen Europa zu adoptieren, es nicht einen einzigen gebildeten Mann im ganzen Reich gibt, der den entferntesten Begriff davon hätte, was europäische Kultur in Wirklichkeit ist. “" Im Folgenden soll durch einige spezifischere zeitgenössische Stellungnahmen aufgewiesen werden, worin die Motive für die Rechtsmodernisierung gesehen und wie die involvierten Rechtskulturen eingeschätzt wurden.

\section{B. Zur Frage der Kenntnis des Anderen}

Als seit Mitte des 19. Jh. die chinesische mit der westlichen Rechtskultur in konfliktreichen Kontakt gerieten, und in der ersten Dekade des 20. Jh. beide Rechtskulturen auch in der Gesetzgebung aufeinanderzutreffen sich anschickten, wussten beide Seiten wenig voneinander. Der vom 16. bis zum 18. Jh. durch die Jesuitenmission erfolgte zweiseitige Kulturtransfer betraf keine Rechtsmaterien. Zwar haben die Missionare neben dem Theologischen auch mathematische, astronomische, kartographische und selbst künstlerische Kenntnisse nach China vermittelt, Informationen zum abendländischen Recht gehörten jedoch nicht zu den Gegenständen, mit denen man bei den damaligen Chinesen auf Interesse gestoßen wäre. Und wenn ihnen auf indirekte Weise solche Informationen vor Augen kamen, so riefen sie unvermeidlich Unverständnis und Kränkung hervor. Jacques Gernet hat in Chine et christianism, action et réaction (Paris 1982) 4 gezeigt, wie durch die Predigt des christlichen Menschenbilds abendländische Rechtsvorstellungen notwendig involviert waren und von den Chinesen ebenso notwendig als subversive Anmaßung empfunden wurden. So untersagten es die Ming- und Qing-Kodizes, dass gewöhnliche Bürger direkt den Himmel anrufen, denn dem Himmel zu opfern war nach chinesischer Auffassung ein Reservat des Kaisers. Die Missionare missachteten chinesische Gesetze auch dadurch, dass sie bekehrte chinesische Männer aufforderten,

2 Zu diesen Vorgängen Robert Heuser, Grundriss der Geschichte und Modernisierung des chinesischen Rechts, Baden-Baden 2013, S. $125 \mathrm{ff}$.

3 Ku Hung-Ming, Chinas Verteidigung gegen europäische Ideen, hrsg. von Ku Neng-yi, Taipei, 1956, S. 66 f. Erst 1848 war das erste chinesische Werk (von Xu Jiyu) über Weltgeschichte erschienen; chinesische diplomatische Vertretungen in westlichen Ländern wurden erst seit den 1870er Jahren eingerichtet. Vgl. allgemein Fang Weigui, „Kulturvergleich und -transfer. Welche Botschaften haben die ersten chinesischen Gesandten vom Westen nach China gebracht?", Orientierungen 1/2004, S. 1 ff. Ein anderer Mitarbeiter Zhang Zhidongs, der Übersetzer westlicher staats- und sozialwissenschaftlicher Klassiker Yan Fu (1853-1921), schrieb gegen Ende des Jahrhunderts: „Chinese understanding of the necessity of learning Western methods did not begin until after the defeat of China by Japan in 1895.“ (Ssu-Yu Teng, John K. Fairbank, China's Response to the West. A Documentary Survey 1839-1923, Cambridge/Mass. 1954, S. 150.

4 Deutsch unter dem Titel Christus kam bis nach China. Eine erste Begegnung und ihr Scheitern, Zürich und München 1984. 
eine ihrer getauften Konkubinen zu heiraten, falls die Ehefrau sich weigerte, christlich zu werden, die Ehefrau also zu verstoßen. Nach chinesischem Recht war es jedoch strafbar, die rechtmäßige Frau zu verstoßen und zu ihrer Lebenszeit eine Konkubine zu heiraten. Gernet berichtet weiter, dass Matteo Ricci seinen chinesischen Schülern erläuterte, sie hätten drei Väter: den Vater im Himmel, den Herrscher und den leiblichen Vater. „Wenn nun“, so zitiert Gernet Ricci, „ein Sohn einem höheren Vater gehorcht und die Befehle eines niedrigeren Vaters missachtet, schadet das der Kindespflicht nicht". Dies beinhaltete eine sehr konfliktträchtige Auslegung der ersten chinesischen Tugend und kam einer Anstiftung zur Rebellion gleich.

Ebenso gehörte umgekehrt das chinesische Recht nicht zu den Gütern, die im Europa des 18. Jh. bekannt und geschätzt wurden. Zwar waren es nicht nur Dinge wie Porzellantechnik, Seidenraupenzucht und Papiertapete, ästhetische Vorstellungen in Gartenbau und Architektur, die in Europa Furore machten, die Aufklärungsgeister des 18. Jh. erörterten auch soziale und politische Institutionen Chinas, wie sie in Berichten von Jesuiten und Kaufleuten beschrieben worden waren. Dabei kam Montesquieu vom Standpunkt seiner gerade entwickelten Gewaltenteilungslehre zu einer skeptischen Beurteilung, wobei sich seine Intuition als verlässlicher erwies als die manch eines schwärmerischen Zeitgenossen. „Für die Gesetzgeber Chinas“, heißt es im 19. Kapitel des 19. Buches des L'esprit des lois, „war das Hauptziel der Regierung die Befriedung des Reichs. Als das geeignetste Mittel dazu erschien ihnen die Unterordnung. Dieser Idee gemäß glaubten sie, man müsse Hochachtung vor den Vätern einflößen. Darauf verwandten sie ihre ganze Kraft. Sie führten unendlich viel Riten und Zeremonien zur Verehrung der Väter während ihres Lebens und nach ihrem Tod ein...Indes waren das nur Teile des gleichen Gesetzbuches, und dies Gesetzbuch war sehr umfassend."

Es ist nicht bekannt, dass dieses Gesetzbuch, der Ming- oder der Qing-Kodex, in einem Jesuitenbericht jemals erwähnt worden ist. Erst dessen Kenntnis würde ein Bild vom chinesischen Recht vermittelt haben. Der britische Diplomat Sir Thomas Staunton, der 1810 die erste Übersetzung der zentralen Teile des Qing-Gesetzbuchs (大清律例) vorgelegt hat, äußerte in seinem Vorwort Bedauern darüber, dass die Jesuiten ,had not been more directed to the objects that were principally desirable“. „As statesmen and philosophers“, fährt er fort, „we have to examine the principles, operations, and consequences of the civil policy, characteristic laws, and general systems of government and constitution, not indeed the best or the purest, but certainly the most anciently, and, if we may judge from its duration, the most firmly established, and the most conformable to the genius and character of the people, of any of which mankind has had experience. ${ }^{* 5}$

Stauntons Übersetzung blieb für mehr als ein halbes Jahrhundert der einzige originale chinesische Rechtstext in einer europäischen Sprache. Joseph Kohlers 1886 in Würzburg erschienene Abhandlung „Das chinesische Strafrecht“ beruht im Wesentlichen auf ihr. 1899 bemerkte der in der chinesischen Zollverwaltung tätige britische Beamte Ernest Alabaster in

5 Ta Tsing Leu Lee, Being the Fundamental Laws, and a Selection from the Supplementary Statutes of the Penal Code of China, London, 1810, S. V, XIII. 
der Einleitung seiner Notes and Commentaries on Chinese Criminal Law, ,that Western aid has not been greatly forthcoming in this branch of sinology“ ${ }^{6}$ So konnte selbst er es als „, höchst fraglich“ bezeichnen, ,ob es vor der Ming-Dynastie einen Kodex im eigentlichen Sinn gegeben habe“, 7 ein Zweifel, von dem ihn auch der im Vorwort dankend erwähnte Cambridger Sinologieprofessor Herbert Giles nicht kurieren konnte. Dessen ungeachtet leistete Alabaster dadurch einen beträchtlichen Beitrag, dass er den Qing-Kodex im Zusammenhang mit wichtigen Gerichtsentscheidungen behandelte. Dabei gelangte er zu einer geradezu überschwänglichen Beurteilung: „The Code is infinitely more exact and satisfactory than our own system, and very far from being the barbarous cruel abomination it is generally supposed to be...In general the Chinese system may be characterized as less Draconian than our own, but in some cases it is more severe...On the whole, the Chinese system both of law and its administration, may be safely regarded with considerable admiration." ${ }^{\text {8 }}$ Es beeindruckte ihn, dass nach dem Qing-Kodex die Strafe für jede Straftat exakt bestimmt ist und dem Richter kein Spielraum bei der Strafzumessung verbleibt, wodurch sich der Kodex „,to any expansion of authority“ als eine „unübersteigbare Schranke“ erweise. Ob dieses sehr positive Urteil auch vor dem Hintergrund damaliger englischer Strafrechtsverhältnisse erklärbar ist, wäre eine Untersuchung wert.

Leider hat Max Weber weder Staunton, noch Alabaster, der sich im Anhang seiner „Notes and Commentaries“ mit Ähnlichkeiten zwischen dem chinesischen ,,and other civilised systems of law" befasste, in seiner die chinesische Wirtschaftsethik betreffenden Studie berücksichtigt ${ }^{9}$, sondern sich - sicher unbeabsichtigt - eher auf solche sinologischen Quellen gestützt, die vom Geist des „Zivilisierungsauftrags“ durchdrungen waren. ${ }^{10}$ Auch wenn sein „Chinabuch“ auf Dauer imponierend bleiben wird, so halten seine Aussagen zu Recht und Justiz im kaiserlichen China einer Überprüfung nicht stand. Etwa wenn er ausführt: „,Der antiformalistische patriarchalische Grundzug verleugnete sich nirgends: anstößiger Lebenswandel wurde gestraft auch ohne Spezialbestimmung. Das Entscheidende war aber der innerliche Charakter der Rechtsfindung: Nicht formales Recht, sondern materiale Gerechtigkeit erstrebte der ethisch orientierte Patrimonialismus hier wie überall. Eine offizielle Präjudiziensammlung fehlte daher, trotz des Traditionalismus, weil der formalistische Charakter des Rechts abge-

6 Notes and Commentaries on Chinese Criminal Law. With Special Relations to Ruling Cases, London 1899, S. XIV f. Etwa gleichzeitig heißt es in Zhang Zhidongs „Chinas Only Hope“(englisch New York etc. 1900, S. 112): „We have met many foreigners who are thoroughly versed in the language and literature of our country, but we have seen few Chinese who know much about Western literature.".

7 Notes and Commentaries, note 7, S. XLI.

8 Ibid., S. LXII ff.

9 Von „Staunton“ heißt es 1907, dass „diese Übersetzung sehr selten ist“, d.h. schwer auffindbar ist (Guiseppe Mazzarella, „Über die Sammlung orientalischer Rechtsquellen“, Blätter für vergleichende Rechtswissenschaft und Volkswirtschaftslehre(1907), S. 264).

10 Vgl. Webers Angaben „Zur Literatur“ in der ersten Fußnote zu „Konfuzianismus und Taoismus“ (Max Weber, Die Wirtschaftsethik der Weltreligionen. Konfuzianismus und Taoismus, Schriften 1915-1920, Studienausgabe, Tübingen 1991, S. 27 f.). 
lehnt wurde und, vor allem, kein Zentralgericht wie in England bestand... Die Justiz blieb demgemäß weitgehend Kadi-Justiz... Dem Patrimonialismus des chinesischen Wohlfahrtsstaates mit seiner schwachen Amtsgewalt (fehlte) der Sinn für die formale Entwicklung des weltlichen Rechts... Vor allem entschied der chinesische Richter, als typischer Patrimonialrichter, durchaus patriarchal,...ausdrücklich nicht nach formalen Regeln. Vielmehr... nach

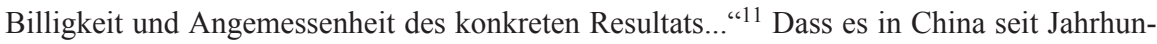
derten weder an formalem Recht, noch an einer Gerichtspyramide mit einer obersten Gerichtsinstanz, noch an amtlichen Präzedenzsammlungen ermangelte und die Tätigkeit der Richter nichts mit dem gemein hat, was als „Kadi-Justiz“ bezeichnet wird, hätte Max Weber jedenfalls für die Qing-Dynastie bereits den erwähnten Werken von Staunton und Alabaster entnehmen können. ${ }^{12}$

\section{Erwartungen an die Rechtserneuerung}

\section{Strategien}

Angesichts der Genese der Rezeptionsvorgänge aus dem Geist der Response to the West ist es naheliegend, dass die Aussicht auf Überwindung der ausländischen Privilegien das erste und auf einen breiten Konsens innerhalb der chinesischen Beamtenschaft stoßende Motiv der Rechtsreform wurde. Shen Jiaben hat diesen Gesichtspunkt in der Absicht besonders hervorgehoben, die konservativen Kreise der Beamtenschaft mit der Rechtsmodernisierung zu versöhnen. So heißt es in seiner Throneingabe von 1905: „Die Strafgesetze der westlichen Staaten wiesen früher mehr Grausamkeiten auf als die Chinas. Während der vergangenen etwa hundert Jahre jedoch wurde das westliche Recht allmählich gemildert, was dazu führte, dass die rigorosen chinesischen Gesetze von Ausländern als inhuman (不仁) eingeschätzt werden. Statt an unseren alten Strafnormen festzuhalten und den Ausländern so eine Vorwand zu liefern, sich den chinesischen Recht nicht zu unterwerfen, sollten wir uns zu Änderungen entschließen, und dabei Gesetze anderer Länder berücksichtigen. " ${ }^{13}$

Die Reformkreise um Shen Jiaben, der sich durch inzwischen vorliegende Übersetzungen westlicher rechts- und staatswissenschaftlicher Abhandlungen, durch aus Japan heimgekehrte chinesische Studenten, zuletzt auch im Kontakt mit japanischen Beratern über westliches, besonders kontinentaleuropäisches Recht und seiner Geschichte - in welchem Umfang auch immer - informiert hatten, erwarteten von der europäisch geprägten Gesetzgebung aber mehr als einen Souveränitätsgewinn. Unter der generellen Prämisse „Wohlstand und Macht des Staates“(富国强兵) zu erringen, ging es ihnen auch um konkrete und durchaus einschneidende

11 Ibid., S. 108 f., $140 \mathrm{f}$.

12 Was Karl Bünger, „Max Webers Ansichten über Recht und Justiz im kaiserlichen China“, Oriens Extremus, 1972, S. 9 ff. und ders., „War China ein patrimonialer Staat?“, Oriens Extremus, 1977, S. $167 \mathrm{ff}$. näher erörtert hat.

13 Übersetzung der Eingabe in Marinus Johan Meijer, The Introduction of Modern Criminal Law in China, Appendix V, Batavia 1949, S. 164. 
Änderungen des überlieferten Rechtssystems: Im Bereich des Privatrechts um die Etablierung des sog. ,Rechte-Standards“ (权利本位), d.h. eines Systems, wonach das Gesetz die subjektiven Rechte der Einzelpersonen und Korporationen schützt ${ }^{14}$ und nicht - wie traditionell allein Pflichten reflektiert, ferner um die Schaffung von dem (internationalen) Handelsverkehr dienlichen Gesetzesnormen. So lautete der erste der beiden Grundsätze, von denen man sich bei der Ausarbeitung des 1911 dem Thron vorgelegten ZGB-Entwurfs hat leiten lassen: „Übernahme der in der modernen Wirtschaftswelt allgemein gebräuchlichen Rechtsregeln.“ Da der Verkehr über die Ozeane immer mehr zunehme, in chinesischen Häfen der Handelskrieg tobe und die Überseechinesen bereits moderne Rechtsformen praktizierten, bliebe China im eigenen Interesse nichts anderes übrig, als die allgemein üblichen Standards zu übernehmen. ${ }^{15}$ Seit dem letzten Drittel des 19. Jh., während dem in den Küstenstädten ein allmählicher Übergang von natural- zu warenwirtschaftlichen Verhältnissen vonstatten ging, waren zunehmend Unternehmen in Erscheinung getreten, die nicht mehr als reine Familieneinrichtungen qualifiziert werden konnten und deshalb ein vorher unbekanntes Bedürfnis nach „Rechtssicherheit" entwickelt hatten, auch der Schutz des Publikums vor Phantasiegesellschaften war als Problem in Erscheinung getreten. ${ }^{16}$ Diese Situation ,verlangte dringend nach neuer Gesetzgebung““ ${ }^{17}$ „Soziale und wirtschaftliche Armut“, heißt es in einer zeitgenössischen Stellungnahme, ,sind das Ergebnis eines unterentwickelten Handels, und dieser Zustand resultiert aus dem Mangel an Gesetzen. “18 Dies erinnert an Montesquieus Bemerkung, wonach „für ein Volk, das sich dem Handel und dem Meer widmet, ein umfangreicheres Gesetzbuch nötig ist als für ein Volk, das sich mit Ackerbau begnügt. “19

Im Bereich des Strafrechts erwarteten die Reformer von der neuen Gesetzgebung neben der Einführung des im alten Recht im Grundsatz, aber unvollkommen entwickelten Legalitätsprinzips und der Humanisierung des Sanktionensystems vor allem eine Neubestimmung des Verhältnisses von Recht (Gesetz) und Moral, d.h. die Reduzierung der strafrechtlichen Sanktion in Richtung auf ein ,ethisches Minimum“. So sollte der ganze Bereich des Ungehorsams innerhalb der Familie nicht mehr strafrechtsrelevant, sondern allein eine Angelegenheit der Erziehung sein. Hier ist es interessant zu sehen, wie Shen in seiner Argumentation bemüht war, die Konservativen um Zhang Zhidong (1837-1909) mit Hinweis auf den ursprünglichen Konfuzianismus des Altertums zu überzeugen. Seit den Zeiten von Han und

14 Vgl. etwa den von Oliver Simon und $L u$ Ye übersetzten Aufsatz aus dem Jahre 1908: „Über die wichtigen Elemente bei der Erstellung von Rechtskodifikationen“, Zeitschrift für Chineisches Recht (2006), S. 373 ff., 376.

15 Yang Honglie, 中国法律发达史, Shanghai, 1930, S. 906.

16 Dies erwähnt auch der gemäßigte Reformer Zhang Zhidong, einer der führenden chinesischen Politiker des Zeitalters, in seinem Aufruf „China’s Only Hope“ (englisch New York etc. 1900, S. 57).

17 So $X u$ Lizhi, , 清末的商事立法及其特点 (Die Handelsgesetzgebung der Späten Qing und ihre Merkmale), in: 法学研究 1989, Nr. 3, S. 89.

18 Zitiert in Zhu Ying, „The Economic Laws and Regulations of the Late Qing“, in: Social Science in China, Summer 1995, S. 123.

19 Vom Geist der Gesetze, 18. Buch, 8. Kapitel. 
Tang und bis in die Gegenwart war es üblich, einen gleichsam automatischen Zusammenhang zwischen Moralverfehlung und Strafsanktion anzunehmen. Eine typische Formulierung dieses Zusammenhangs lautet: „Handlungen, welche die Regeln der Sittlichkeit verletzten, werden von Strafe erfasst; die Regeln der Sittlichkeit zu verlieren heißt, in den Bereich der Strafen zu gelangen“(礼之所去，刑之所取，失礼即入刑) ${ }^{20}$. Shen vertrat nun die Ansicht, dass ein solcher Automatismus dem ursprünglichen Konfuzianismus - Lunyu, Mengzi - fremd war, der Bereich der strafbaren Handlungen also eingeengt werden müsse. Lunyu und Mengzi hatten in der Tat die Schaffung von Ordnung durch weitgehend unsanktionierte Sittlichkeitsregeln (礼) propagiert. Ironischerweise verhielt es sich also so, dass die Konservativen dem nichtkanonischen Xunzi nahe standen, während die Modernisierer für sich in Anspruch nahmen, näher bei Menzius zu sein. Shen suchte nun den Eindruck zu erwecken, dass das moderne westliche Strafrecht eine größere Nähe zum ursprünglichen Konfuzianismus aufweise als das gegenwärtig geltende chinesische Recht. ${ }^{21}$ Hier zeigt sich das Charakteristische an Shens Reformansatz, nämlich die Auffassung, dass Modernisierung in der frühen chinesischen Tradition wurzeln könne, ein Ansatz, der später durch utilitaristische Erwägungen aus der Sicht der Parteipolitik überlagert wurde oder gänzlich aus dem Blick geriet.

\section{II. , ,Volksgeist“ und Gewohnheitsrecht}

Die Reformer hegten wohl auch die Vorstellung, indigenes Recht in die Kodifikation einfließen zu lassen. So wird der 1911 veröffentlichte Entwurf eines ZGB mit einem Paragraphen eingeleitet, der eine bekannte Vorschrift des Schweizer ZGB von 1907 variiert: „Ist eine Zivilangelegenheit in diesem Gesetz nicht geregelt, so ist Gewohnheitsrecht (习惯法), besteht kein Gewohnheitsrecht, so sind die allgemeinen Rechtsgrundsätze (法理) maßgebend“. Dem Gewohnheitsrecht wurde aber nicht nur der Rang einer sekundären Rechtsquelle beigemessen, sondern man war sich auch bewusst, dass seine Erforschung für die Privatrechtsgesetzgebung überhaupt von Bedeutung ist. Dies ergibt sich auch aus dem zweiten Grundsatz, von denen man sich bei der Ausarbeitung des ZGB-Entwurfs hat leiten lassen: Berücksichtigung solcher Regeln, die dem Volksempfinden (民情) gemäß sind. Denn: „Die die menschlichen Angelegenheiten betreffenden Gesetze entstehen aus dem Volksgeist und den Gewohnheiten (人事 法缘于民情风俗而生), man kann ihnen nicht durch Zwang Geltung verschaffen, andernfalls würde man uns zu Recht vorwerfen, wir verhielten uns wie jemand, der versucht, Zehen den Schuhen anzupassen. “22 Ob der insoweit an die moderne Gesetzgebung gerichteten Erwartung - Berücksichtigung eigener Rechtsgewohnheiten in der neuen Gesetzgebung - ausreichend entsprochen wurde, kann bezweifelt werden. Andererseits ist die warnende Metapher von den Zehen, die den Schuhen angepasst werden, einer der zu dieser Zeit ganz seltenen Hinweise

20 Chronik der Jin-Dynastie, Strafrechtskapitel (晋书刑法志).

21 Kenneth G. Wheeler, Shen Jiaben (1840-1913): Toward a Reformation of Chinese Criminal Justice, Yale University Dissertation, 1998, S. 206 ff.

22 Yang Honglie, ibid., S. 907. 
auf das Problem der Adaptabilität von Rechtsimporten. Ein anderer betrifft die ominöse Stellungnahme eines 1909/10 an der Deutsch-Chinesischen Hochschule in Qingdao tätigen deutschen Rechts-Dozenten namens Harald Gutherz. Die von ihm geäußerte Ansicht, dass die "von den Gelehrten des Altertums geschaffenen Gesetze China perfekt" seien und es nur nachteilig sei, ,sie durch ausländische zu ersetzen“, wird in der chinesischen Literatur als reiner Opportunismus eines Ausländers, der sich bei den Konservativen andienen wollte, abgetan. Dass Shen Jiaben über diesen Einwand des Ausländers sich höchst ungehalten zeigte, macht deutlich, wie ernst es ihm damit war, grundlegende Veränderungen der chinesischen Rechtstradition $\mathrm{zu}$ erreichen. ${ }^{23}$

\section{Modellwahl}

Wie das Adaptionsproblem, so wurde auch die Frage nach der Modellwahl - kontinentaleuropäisches oder englisches Rechtssystem - kaum aufgeworfen. Offenbar war mit dem Vorläufer Japan alles geklärt. Zur Frage der Orientierung am kontinental-europäischen und dabei am deutschen Modell kann man aber mit der neueren Literatur dem Geist der Zeit drei Argumente entnehmen: Bewährung, Qualität und Macht. Nach dem Bewährungsargument hat die Übernahme deutschen Rechts in Japan gezeigt, dass das Ziel, die ungleichen Verträge aufzuheben, rasch erreicht wurde. Shen Jiaben wird mit den Worten zitiert: „Das alte japanische System hatte viel vom Tang-Recht (唐法) übernommen; seit der Meiji-Restauration nutzt man westliches Recht (西法), und Japan wurde binnen weniger Jahrzehnte zu einem starken Staat.“Zum Qualitätsargument wird darauf verwiesen, dass die Qing-Reformer im deutschen Recht die damals modernste Zivilrechtskodifikation - vor allem erkennbar an der Existenz eines Allgemeinen Teils - geschätzt hätten. Zum Machtargument wird vorgebracht, es sei den Qing-Reformer deutlich gewesen, dass Deutschland nach Gründung des Deutschen Reichs und der damit einhergehenden Gesetzgebung eine rasche Entwicklung in Wirtschaft, Wissenschaft und Technik genommen hatte. Das Recht hatte sich somit als Mittel der Stärkung des Staates erwiesen. ${ }^{24}$

Die Hinwendung zu einem civil law System war jedoch mehr der Hast und Hektik geschuldet, mit der die Entwurfsarbeit vonstatten ging, als tiefschürfender Erwägung des Pro und Contra bezüglich einer Wahl zwischen den beiden westlichen Rechtskreisen. Vereinzelt gab es Stimmen, die sich dahingehend äußerten, dass eine grundsätzliche Hinwendung zum case law System eine intensivere Rechtsmodernisierung aus eigenen Ressourcen und damit größere Nachhaltigkeit ermöglicht hätte. So wurde schon früh aus Kreisen chinesischer USAStudenten die Ansicht geäußert, dass das case law System den chinesischen Verhältnissen

23 So bei Wang Boqi, 近代法律思想与中国固有文化 (Modernes Rechtsdenken und angestammte chinesische Kultur), Taibei, 1956, S. $16 \mathrm{ff}$.

24 Wang Limin, ,论清末德国法对中国近代法制形成的影响“ (Der Einfluss des deutschen Rechts auf die Gestaltung des modernen chinesischen Rechtssystems in der späten Qing-Zeit), in: 上海社会科 学院学术季刊, 1996, Nr. 2, S. 132 ff. 
besser entspricht: ${ }^{25}$ „,The civil part of the law is not embodied in any (traditionel Chinese R.H.) code. The magistrate bases his decision partly on precedent and partly on some principle of right and wrong. In theory it is exactly like the case system, and is the way in which the English common law has developed." Da die Gewohnheiten in den diversen Provinzen sehr unterschiedlich seien, sei eine landesweit maßgebliche Kodifikation auf solcher Grundlage nicht möglich. Der Schwerpunkt der Reform müsse auf das Verfahrensrecht gelegt werden, um das chinesische Recht zu befähigen, die Vorteile des Fallrechts-Systems zur vollen Entfaltung zu bringen. Wörtlich heißt es: „The low state in our administration of justice is largely due to the lack of good procedure in Chinese law rather than to the principles of law. To correct the defect three things need to be done. There should be a set of scientific rules of pleading and evidence; lawyers should be admitted to the court to argue the case and enable the judge to find and apply the law; the judge's decision must be written out and made public. If these three conditions are kept, a foundation is laid for the development of Chinese law as a native growth and not as an extraneous graft. In the meantime the law schools in China, instead of studying Japanese text-books law, should study scientifically the records of cases decided in the Chinese courts from the earliest times downward with the view to discovering the principles of Chinese law.“

Auch in China tätige englische Kaufleute haben schon früh beobachtet, dass die Chinesen über ein auf Gewohnheit und Fallrecht beruhendes Handelsrecht verfügen, durchaus vergleichbar ,with the commercial law of other countries, which (and especially that of England) is formed much more upon custom and the necessities of individual cases than upon abstract logical principles. ${ }^{\text {26 }}$ Der Autor entnimmt seiner Geschäftspraxis die Erkenntnis, dass dem chinesischen Recht die Prinzipien des Kauf- und Werkvertrags, der Bürgschaft, der Gesellschaft u.a. vertraut seien, „certain broad principles of commercial law are generally established among the Chinese", und es an dem verbreiteten Vermittlersystem, den Compradoren, liege, dass die ausländische Kaufmannschaft nur limitierte Kenntnisse des chinesischen Handelsrechts erlangten. Der Autor weist jedoch darauf hin, dass ,the science of Law in China has stopped short at a certain point, and there are, therefore, no treaties carefully setting forth the main principles of law and the deductions to be made from them, such as we find in other places“, was der Autor auf die traditionelle Geringschätzung der gebildeten Schichten gegenüber Angelegenheiten des Handels zurückführt. Die neuere Sinologie hat die Beobachtung der zitierten englischen Praktiker bestätigt, indem sie auf der Grundlage von Vertragsdokumenten aus dem 18. und 19. Jh. zeigt, ,that certain customary laws..., which became more widespread after the 17 th century, enabled families to organize and use resources more efficiently..., and facilitated a variety of property rights exchanges. “27

25 F. Chang, „The Bearing of the Case and Code Law on the Development of Future Chinese Law“, The Chinese Students' Monthly, (1915/16), S. $547 \mathrm{ff}$.

26 A. C. D., Notes on Chinese Commercial Law, The China Review (1873/74), S. $144 \mathrm{ff}$.

27 Fu-mei Chang Chen, Customary Law and the Economic Growth of China During the Ch'ing Period, Ch'ing-Shih Wen-Ti, November 1976, S. 1 ff. 
Ob Großbritannien, als es 1902 sein Versprechen abgab, den chinesischen Rechtsreformbemühungen ,every assistance“ zuteil werden zu lassen, sich vorstellte, China könnte einen Weg des Fallrechtssystems (weiter) beschreiten, scheint daher nicht völlig ausgeschlossen. So hatte man in Hong Kong unter kolonialistischem Vorzeichen das englische Rechtssystem - wie intensiv auch immer - etabliert. ${ }^{28}$ Jedoch erwies sich das Vorbild Japans als so dominant $^{29}$, dass einer Erörterung einer nichtkodifizierenden oder auf eigenen Ressourcen beruhenden Rechtsmodernisierung nicht näher getreten wurde..$^{30}$

\section{C. „Vorauseilende Gesetzgebung“ und lokales Verstehen}

Die Strategie bestand vielmehr darin, zunächst möglichst schnell ${ }^{31}$ die Grundausstattung eines modernen Rechtssystems präsentieren zu können - Kodifikation und Justizorganisation -, um den Zustand der Konsulargerichtsbarkeit zu überwinden. Die zugrunde liegende Denkweise war also durchaus utilitaristisch und nicht getragen von kulturvergleichenden Überlegungen oder von Einsichten in das den westlichen Straf- und Zivilgesetze zugrundeliegende Prinzip der Autonomie des Individuums. Im Übrigen ging man davon aus, dass es sich bei der Rechtserneuerung um, wie man später sagte, ,vorauseilende Gesetzgebung“(超越立法) handelte, in der man ein Muster sah, dem sich der Sozialprozess und das Rechtsbewusstsein allmählich durch die Geschäfts- und Gerichtspraxis einformen werden, was als zweiseitiger Prozess begriffen wurde: Sollten Sozialleben und Rechtsbewusstsein sich durch das Gesetz formen lassen, so hatte das Gesetz seinerseits sozialkonform interpretiert zu werden. So heißt es in der Einleitung der englischen Ausgabe des ZGB von 1929/30: „Das Gesetzbuch lenkt die Tätigkeit der Bürger in einer Weise, dass sie sich zum Vorteil der Gemeinschaft, deren Teil der

28 Vgl. Berry Fong-Chung Hsu, The Common Law System in Chinese Context. Hong Kong in Transition, Armonk and London 1992. Chi Kuen Lau, Hong Kong's Colonial Legacy, Hong Kong 1997, erörtert S. 130 ff. die Frage: „A Rootless Legal System?“.

29 Zhang Zhidong ging gar davon aus, die Kenntnis des Japanischen könne das Erlernen westlicher Sprachen überflüssig machen. In seinen Aufruf „Chinas Only Hope“ (englisch New York etc. 1900) führte er auf S. 113 aus: „The Japanese have made important selections from all the books of the West and translated them into their own language. By learning Japanese we can possess ourselves of this store of information without troubling about Western languages.".

30 Auch in Japan war der Gedanke der Herausbildung eines Gesetzbuches aus autochthonem Material kaum zum Tragen gekommen. Was Kenzo Takayanagi, „A Century of Innovation: The Development of Japanese Law, 1868-1961“, in: Arthur T. von Mehren, Law in Japan. The Legal Order in a Changing Society, Cambridge/Mass. und Tokyo, S. 24 dazu ausführt, lässt sich auch auf China übertragen: „A common-law lawyer who, like (John) Wigmore, had studied the judicial law of feudal Japan might suggest the possibility of drafting a modern civil code out of the old materials of the indigenous law. The Meiji reformers never thought of such a possibility and would have been unequal to such a task even if they had. Moreover, the unification of law and the abolition of exterritoriality were political exigencies of the first order and allowed of no delay. The reformers, therefore, thought of a quicker way, the importation of a finished legal product...".

31 In Japan war bereits 1869 ein fremdsprachenkundiger Beamter der Zentralregierung, Mitsukuri Rinsho (1846-1897), damit beauftragt worden, „schnell, wenn auch nicht immer ganz genau“ den Code Civil ins Japanische zu übersetzen. Takayanagi, ibid. 
Einzelne ist, auswirkt. Dadurch unterscheidet sich das neue Gesetzbuch sowohl von den individualistischen Gesetzbüchern Europas und Amerikas, als auch von den Gesetzen des Familien-Typs im alten China." Ebenso äußerte sich Fu Bingchang, der Vorsitzende des Zivilrechtsausschusses: „Der neuen chinesischen Gesetzgebung kommt ein bewusst sozialer Charakter zu. Die Lehre der Guomindang sieht die Menschen nicht als unabhängige, isolierte Wesen, sondern in ihren Beziehungen zu der von ihnen gebildeten Gesellschaft. Sie weist ihnen nur insoweit Rechte und Pflichten zu, als die Ausübung solcher Rechte und Pflichten dem friedlichen und geordneten Fortschritt der Gesellschaft dienlich ist. “32

Dies reflektiert die Ideologie der „Drei Volksprinzipien“ (三民主义), die die seit 1927 die politische Macht ausübende Guomindang der Rechtsmodernisierung im Sinne der Adaption oder Akkulturation der neuen Gesetze unterlegt hatte. Es war der in Japan ausgebildete Jurist und Präsident des Nanjinger Gesetzgebungsamts Hu Hanmin (1879-1936), der die „Überleitung“ der „Drei Volksprinzipien“ in die Rechtstheorie vornahm. ${ }^{33}$ Dabei betonte er besonders das „Prinzip des Zusammenhangs von Rechten und Pflichten“(权利义务原则), wonach Rechte nur in dem Maße Anerkennung finden könnten, wie gegenüber der Gesellschaft Pflichten erfüllt würden. Hu ordnete die Gesetzgebung der „Drei Volksprinzipien“ zwischen dem alten chinesischen und dem westlichen Recht ein. Der Unterschied gegenüber dem alten Recht liege darin, dass dieses auf der Basis des Klansystems (家族) beruhte, die monarchische Autokratie schützte, die wirtschaftlichen Verhältnisse einer bäuerlichen Familiengesellschaft im Blick hatte und das Privatrecht im öffentlichen Recht aufgegangen sei, während die Sanminzhuyi-Gesetzgebung auf der Basis der Interessen der Nation (民族) beruhe, den Bedürfnissen einer aus Landwirtschaft und Industrie bestehenden Volkswirtschaft entspreche und zwischen Privat- und öffentlichem Recht unterscheide. Was den Unterschied gegenüber der modernen Gesetzgebung des kapitalistischen Auslands betrifft, so sei dort das Individuum Grundlage der Gesetzgebung. Diesen Rechtsordnungen ginge es vornehmlich darum, die Rechte und Freiheiten der Individuen voneinander abzugrenzen. Hu ist der Ansicht, dass eine solche das Individuum zum Maßstab nehmende Gesetzgebung (个人本位) noch rückständiger sei als die Gesetzgebung des alten China mit ihrem Familien-Standard (家庭 本位). Daher sei die auf den „Drei Volksprinzipien“ beruhende Gesetzgebung mit ihren Standards Gesellschaft, Volk und Staat nicht nur der Gesetzgebung des Familismus, sondern auch der des Individualismus überlegen.

Hier sollte das moderne Recht eine Adaption dadurch erfahren, dass es in den Dienst der Guomindang-Ideologie gestellt wurde. Die tatsächlichen Probleme einer durch die Rezeption ausländischen Rechts aufgegebenen Anpassung an die chinesische sozio-kulturelle Umwelt

32 Fu Binchang, ,新民法与社会本位“ (Neues Zivilgesetz und Gesellschaftsstandard), in: He Qinhua, Li Xiuqing (Hrsg.), 民国法学论文精粹精粹 (Rechtswissenschaftliche Abhandlungen der Republikzeit), 3. Bd., Beijing 2004, S. 26 ff.

33 Nach Chun Yang, ,略湖汉民之立法主持活动“ (Zu Hu Hanmins Tätigkeit der Leitung der Gesetzgebung), in: 法学评论, 2000, Nr. 6, S. 152 ff. 
wurden später präzise in folgenden fünf Widersprüchen, man kann auch von einem fünffachen Paradigmenwechsel sprechen, benannt: ${ }^{34}$

(1) Der Konflikt zwischen dem Konzept der Herrschaft durch Menschen (人治), wonach die Kaisermacht vergötzt wird „der Fürst wertvoll, das Volks verächtlich ist“ und dem Konzept moderner Gesetzesherrschaft (现代法治) mit seiner Forderung, den Staat durch Gesetze zu leiten (依法治国).

(2) Der Konflikt zwischen einem Rechtskonzept, das sich in Strafsachen und Disziplinierung erschöpft und dem modernen auf Güteraustausch und Vertragsschutz gerichteten Rechtskonzept.

(3) Der Konflikt zwischen einer Anschauung, die das Rechtssystem vom Begriff der Pflicht her begreift und die Staatsinteressen zum Maßstab nimmt und der modernen, auf Volkssouveränität gegründeten, durch ein ausbalanciertes Verhältnis von Pflichten und Rechten gekennzeichneten Rechtsanschauung.

(4) Der Konflikt zwischen dem Hierarchie-Konzept des patriarchalischen Sippensystems (宗 法家族) mit Ehe und Blutsverwandtschaft als Grundlage und dem Gleichheitskonzept des modernen Rechtssystems.

(5) Der Konflikt zwischen einem ethischen System, das auf verinnerlichten Moralgeboten basiert, und dem Prozessbewusstsein des modernen Rechtssystems.

In diesen Gegenüberstellungen schlägt sich eine fundierte und nicht mehr durch ideologische Vorgaben - seien sie konfuzianischer, sunyatsenischer oder marxistischer Natur - verzerrte Kenntnis sowohl der westlichen wie der eigenen Rechtskultur nieder. Die Adaption des rezipierten Rechts stellt sich im Wesentlichen als Auseinandersetzung mit diesen Widersprüchen dar. Da der Paradigmenwechsel aus der eigenen Kultur erwachsen und deren Rahmen eingepasst sein muss, wird aus dem rezipierten Recht nicht ein „verwestlichtes“ Rechtssystem geformt, sondern es entsteht etwas Neues, das als local knowledge (Clifford Geertz) erkennbar und erfahrbar sein wird.

34 Nach Li Qirui, 中国传统法律文化变革我见 (Meine Ansicht zum Wandel der traditionellen chinesischen Rechtskultur), in: 法律科学 1995, Ergänzungsausgabe, S. 96 ff. So schon in Robert Heuser, Einführung in die chinesische Rechtskultur, Hamburg 1999, S. 158 f. 